\title{
STUDIES ON THE EFFECTS OF CAPTOPRIL ON RATS' RENAL ISCHEMIA REPERFUSION (I / R) INJURY MEDIATED AND THE MECHANISM OF ACTION
}

\author{
Z. Xiao \\ Hunan Children's Hospital, Changsha, China
}

Objective: To investigate the mechanism of renal I / R injury and its key factors, and analyze how angiotensin-converting enzyme inhibitor (captopril) have protective effect on renal I / R injury.

Methods: In this study, I / R model was made through ligating one side renal vessel. Renal function indexes including Scr and BUN were detected through biochemical analysis; renal tissue and the corresponding protein expression were observed by pathomorphology and immunohistochemistry. ELISA detection was used to determined inflammatory cytokines IL- 6 and TNF- $\alpha$ in renal tissue and serum. IL-1 $\beta$, TNF- $\alpha$ expression and activation of MAPK signaling pathway were analyzed by RT-PCR and Western blot respectively. Turbidimetry was applied to essay serum complement.

Results: After I / R for $1 \mathrm{hr}$, Scr and BUN value started to increase, and presented significantly after $2 \mathrm{hr}$.

BUN and Scr values were decreased markedly after treatment of renal I / R injury with captopril, inflammatory cell infiltration in renal glomeruli and the level of inflammatory cytokines IL-6 were significantly reduced, and swelling of tubular epithelial cells on the proximal and juxtamedullary regions was decreased. After treatment with captopril, the JNK, ERK and P38 phosphorylation was still high, no significant difference with I / R group.

Conclusion: Renal I / R injury can lead to increased expression of pro-inflammatory cytokines, and activate MAPK signaling pathway. Captopril affects the activation of complement system rather than MAPK inflammatory signaling pathway, and thus lessens I / R injury of renal tissue through inhibiting expression of some inflammatory cytokines. 